\title{
Compact coherent anti-Stokes Raman scattering microscope based on a picosecond two-color Er:fiber laser system
}

\author{
Günther Krauss, ${ }^{1}$ Tobias Hanke, ${ }^{1}$ Alexander Sell, ${ }^{1}$ Daniel Träutlein, ${ }^{1}$ Alfred Leitenstorfer, ${ }^{1}$ \\ Romedi Selm, ${ }^{2}$ Martin Winterhalder, ${ }^{2}$ and Andreas Zumbusch ${ }^{2, *}$ \\ ${ }^{1}$ Department of Physics and Center for Applied Photonics, University of Konstanz, D-78457 Konstanz, Germany \\ ${ }^{2}$ Department of Chemistry and Center for Applied Photonics, University of Konstanz, D-78457 Konstanz, Germany \\ *Corresponding author: andreas.zumbusch@uni-konstanz.de
}

Received June 30, 2009; revised August 7, 2009; accepted August 17, 2009; posted August 27, 2009 (Doc. ID 113630); published September 14, 2009

We present a compact coherent anti-Stokes Raman scattering microscope based on a widely tunable picosecond Er:fiber laser. Intense and bandwidth-limited $1 \mathrm{ps}$ pump pulses at a center wavelength of $775 \mathrm{~nm}$ are generated via frequency mixing within the broadband fundamental at $1.55 \mu \mathrm{m}$. Narrowband Stokes pulses are obtained by frequency shifting of solitons in a highly nonlinear bulk fiber and subsequent secondharmonic generation. The tuning range from $850 \mathrm{~nm}$ to $1100 \mathrm{~nm}$ gives access to vibrational resonances between $1150 \mathrm{~cm}^{-1}$ and $3800 \mathrm{~cm}^{-1}$. A first imaging application in the spectral region of $\mathrm{CH}$ stretch vibrations is demonstrated. (C) 2009 Optical Society of America

OCIS codes: $300.6230,190.7110,140.3510,140.3500,170.5810 .$.

Coherent anti-Stokes Raman scattering (CARS) microscopy is a novel type of vibrational microscopy. It allows the creation of molecular selective contrast without the need for external labeling. Therefore it recently stirred much interest for biomedical applications [1-3]. In order to obtain a CARS signal it is necessary to temporally and spatially overlap two pulses with frequencies $\omega_{P}$ and $\omega_{S}$. With tight focusing using high numerical aperture objectives, the CARS signal is generated efficiently in a collinear excitation geometry, and high three-dimensional spatial resolution is readily achieved [4]. If the frequency difference between pump and Stokes, $\omega_{P}-\omega_{S}$, matches a Ramanactive resonance, a blueshifted and intense antiStokes signal is emitted at $\omega_{A S}=2 \omega_{p}-\omega_{s}$. Ideally, the linewidth of the used pump laser matches the typical width of vibrations in condensed phase, i.e., approximately $10 \mathrm{~cm}^{-1}$. The most straightforward implementations of CARS microscopy are therefore based on excitation with picosecond pulses of relatively small bandwidth. Existing two-color picosecond systems are, however, expensive and too complicated to be operated in a nonspecialist environment [5-8]. In order to decrease the complexity of the excitation laser system, several approaches based on fiber laser technology have been proposed [9-11]. These systems are working with broadband femtosecond excitation and spectral filtering of the emission. In a different approach, picosecond Yb:fiber lasers have recently been used to pump an optical parametric oscillator in CARS microscopy [12].

In this Letter, we present a two-color picosecond Er:fiber laser that was developed as a compact, costeffective, and highly stable light source for CARS microscopy (Fig. 1). A mode-locked Er:fiber oscillator [13] at a repetition rate of $40 \mathrm{MHz}$ provides seed pulses for two parallel femtosecond amplifier stages [14]. Each Er:amplifier is bidirectionally pumped with two $980 \mathrm{~nm}$ laser diodes of an output power of
$750 \mathrm{~mW}$. In a single pass through the amplifier, the average power of the femtosecond pulse train increases to $330 \mathrm{~mW}$ for each branch, corresponding to a pulse energy of $8.25 \mathrm{~nJ}$. Because the pulses of both amplifiers originate from the same seed source, they are synchronized with attosecond precision [15]. In order to achieve maximum stability as well as turnkey operation, the system operates exclusively with polarization-maintaining components. After the amplifiers, the pulses are coupled out to free space and compressed to a minimum duration of $130 \mathrm{fs}$ in an adjustable silicon prism sequence.

The first amplifier branch is designed to deliver the pump pulses for the CARS process. In order to match typical dephasing times of molecular vibrations in the liquid phase [16], we are aiming at bandwidthlimited transients with a duration of 1 ps. To this end, the amplifier output is focused into a periodically poled lithium niobate (PPLN) crystal for secondharmonic generation. High conversion efficiencies close to $30 \%$ are achieved owing to sum frequency mixing of spectral regions symmetric to the center wavelength of the fundamental [17]. The optimum crystal length for generation of 1 ps pulses is calcu-

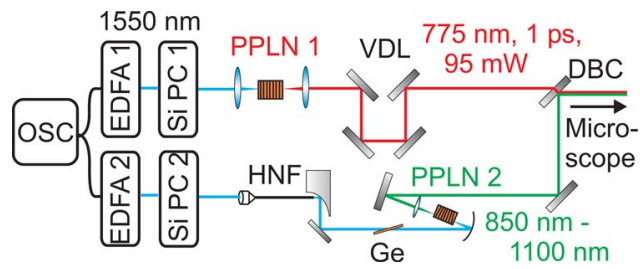

Fig. 1. (Color online) Schematic setup of the laser system. OSC, fs-Er:fiber oscillator; EDFA, erbium-doped fiber amplifier; Si PC, silicon prism compressor; HNF, highly nonlinear germanosilicate fiber for frequency conversion; Ge, $50-\mu \mathrm{m}$-thick germanium plate for dispersion management (optional); PPLN, periodically poled lithium niobate crystal; VDL, variable delay line; DBC, dichroic beam combiner.

(C) 2009 Optical Society of America 
lated to be $10 \mathrm{~mm}$ [18]. Spectra of the pump pulses and the corresponding nonlinear autocorrelation trace are depicted in Fig. 2. The form of the autocorrelation is approximately triangular, indicating a rectangular pulse shape with slightly smoothed flanks. Therefore, we deduce a pulse duration equal to the FWHM of the autocorrelation trace of $1 \mathrm{ps}$. The spectral width of the pump amounts to $11 \mathrm{~cm}^{-1}$. We achieve an average output power of $95 \mathrm{~mW}$ corresponding to a pulse energy of $2.4 \mathrm{~nJ}$ at $775 \mathrm{~nm}$. The pulse durations are about 5 times shorter than that of commercial OPOs used for CARS microscopy, which leads to more efficient signal generation.

In the second branch (see Fig. 1) we generate tunable Stokes pulses. For a first frequency conversion step, the compressed amplifier output is coupled into an assembly of a standard telecom fiber spliced to a highly nonlinear fiber. In this way, the fundamental pulse at $1.55 \mu \mathrm{m}$ is split into a dispersive wave shifting towards the visible and a solitonic part at longer wavelengths [19]. By varying the input chirp of the driving pulses by translating one prism in the $\mathrm{Si}$ compressor we can tune the center wavelengths of these two components [20]. Compared to OPOs, the wavelength of the Stokes pulse can be changed much faster in this way. In order to obtain narrowband radiation with high power spectral densities we frequency double the soliton part of the tunable supercontinuum in a 12-mm-long PPLN crystal with fanout grating design [21]. By adjusting the center frequency of the soliton and the position of the PPLN, the Stokes wavelength is tuned from $850 \mathrm{~nm}$ to 1100 $\mathrm{nm}$ (Fig. 3). Spectral changes of several hundred wavenumbers are achieved solely by changing the PPLN position. Since the soliton from the highly nonlinear germanosilicate fiber is almost bandwidth limited with a slightly negative chirp, no dispersion compensation is necessary before focusing into the second-harmonic generation crystal for target wavelengths from $850 \mathrm{~nm}$ to $950 \mathrm{~nm}$. Lithium niobate ex-
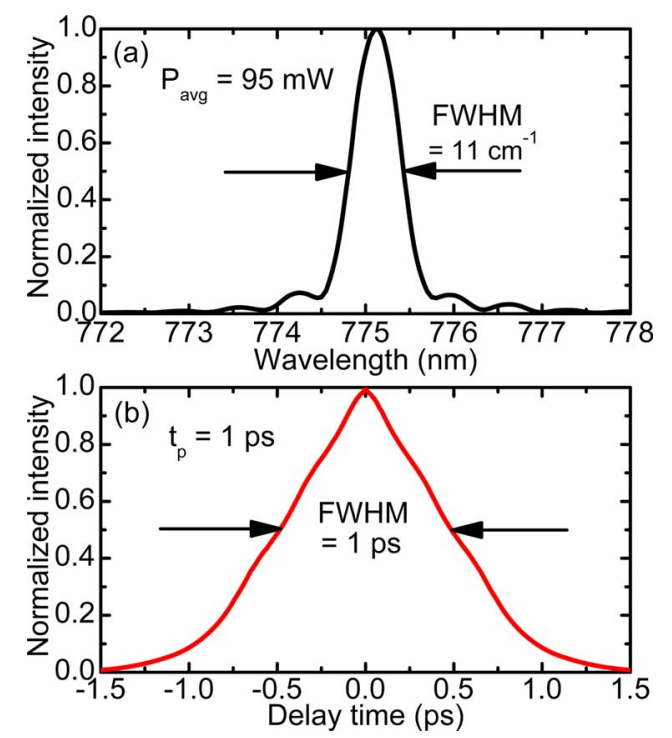

Fig. 2. (Color online) Spectral intensity of the picosecond pump pulses versus wavelength (a) and nonlinear autocorrelation trace (b).

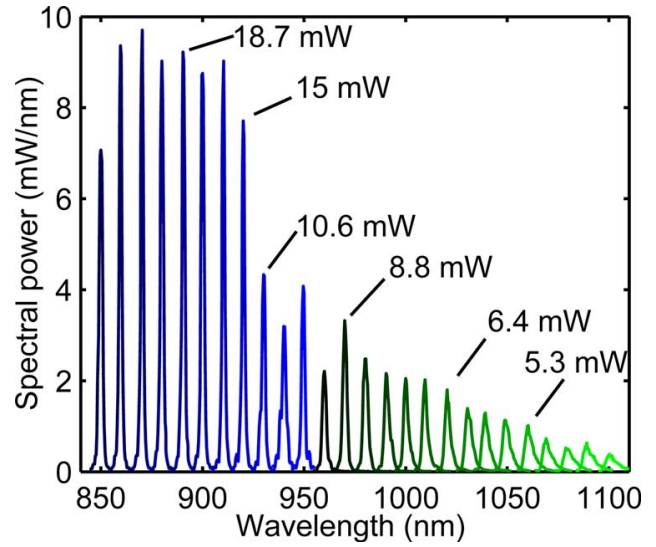

Fig. 3. (Color online) Spectral power of the tunable Stokes pulses versus wavelength. The spectra from $960 \mathrm{~nm}$ to $1100 \mathrm{~nm}$ are generated using a 50- $\mu \mathrm{m}$-thin Ge Brewster plate for dispersion management before frequency doubling. Average output powers at various wavelength positions are indicated.

hibits a zero crossing of the group velocity dispersion at $1900 \mathrm{~nm}$. Therefore, an additional dispersive element has to be included for optimum efficiency of generation of wavelengths longer than $950 \mathrm{~nm}$. We use a $50-\mu \mathrm{m}$-thin freestanding Ge window under the Brewster angle in order to induce the correct prechirp before the PPLN. Over the full Stokes beam tuning range we obtain narrowband emission with average powers between $2.7 \mathrm{~mW}$ and $18.8 \mathrm{~mW}$ (Fig. $3)$.

In order to demonstrate CARS microscopy with the new excitation source, we imaged a mixture of polystyrene (PS) beads (6 $\mu \mathrm{m}$ diameter) and polymethylmetacrylate (PMMA) beads (1-10 $\mu \mathrm{m}$ diameter), which were deposited on a glass cover slip. PS contains both aromatic and aliphatic $\mathrm{CH}$, whereas PMMA contains only aliphatic $\mathrm{CH}$. Thus, both types of beads can be distinguished based on their vibrational spectra as shown in Fig. 4. To date, CARS microscopy has mainly been applied in biological investigations. These are also possible with the laser source presented here, as is demonstrated with the imaging of lipid distribution in live C. elegans [Fig. 4(c)]. In both cases, the Stokes wavelength is combined with the pump pulses in a dichroic beam splitter and the temporal overlap between the two pulses is controlled by a variable delay line. The sample is raster scanned in the focus of a home-built sample scanning microscope employing an $x y z$ piezo stage. Excitation light is focused with an air objective (40×, NA 0.7). The CARS emission is collected in transmission with a second air objective (10×, NA 0.4). Excitation light is filtered out with a combination of three short-pass filters, and the signal is detected with an avalanche photodiode. It should be noted that, apart from its simplicity and low price, the fiber laser used here offers two distinct advantages over commercially available optical parametric oscillator based picosecond laser systems. These are fast tunability and pulse lengths of $1 \mathrm{ps}$, which ideally match the width of vibrations. Whereas commercial systems have pulse durations of $5 \mathrm{ps}$ and longer, the shorter pulses used here drive the CARS process most effi- 

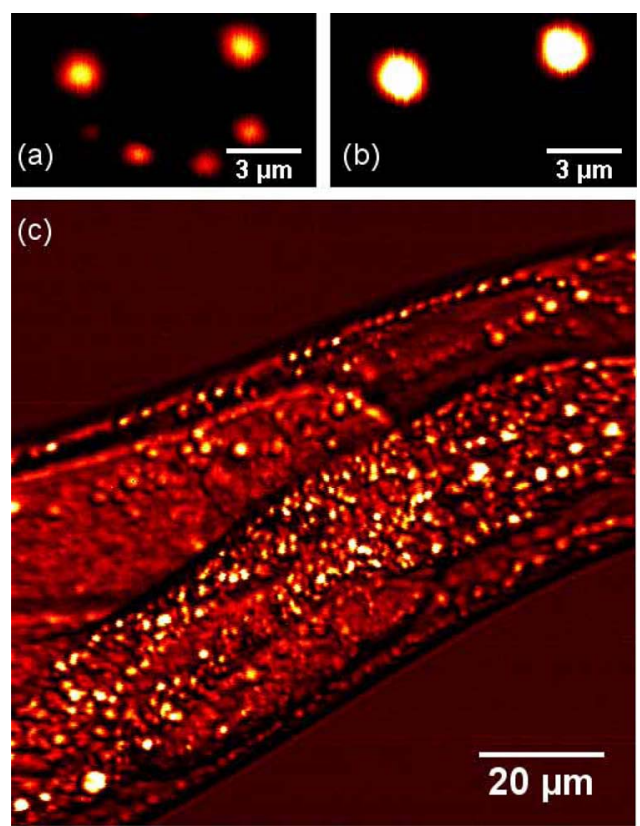

Fig. 4. (Color online) (a) and (b) Microscopic CARS image of a mixture of PS and PMMA beads on a glass slide. (a) Imaging of the aliphatic $\mathrm{CH}$ stretch vibration resonance at $2950 \mathrm{~cm}^{-1}$ and (b) the aromatic $\mathrm{CH}$ stretch vibration at $3050 \mathrm{~cm}^{-1}$. (c) Imaging of the lipid distribution in live C. elegans at $2845 \mathrm{~cm}^{-1}$.

ciently while still offering the best possible spectral resolution.

In conclusion we have demonstrated a turn-key and compact laser source for picosecond CARS microscopy based entirely on telecom-compatible Er:fiber technology. Intense pump pulses at $775 \mathrm{~nm}$ and widely tunable Stokes pulses are generated. This system allows to probe vibrational transitions with frequencies between $1138 \mathrm{~cm}^{-1}$ and $3812 \mathrm{~cm}^{-1}$. The pulse energies of $2.4 \mathrm{~nJ}$ in the pump and up to 0.47 $\mathrm{nJ}$ in the Stokes branch render fast scanning implementations readily accessible.

This work has been supported by Carl Zeiss AG and TOPTICA Photonics AG.

\section{References}

1. A. Volkmer, J. Phys. D 38, R59 (2005).

2. M. Müller and A. Zumbusch, ChemPhysChem 8, 2156 (2007)

3. C. L. Evans and X. S. Xie, Annu. Rev. Anal. Chem. 1, 883909 (2008).

4. A. Zumbusch, G. R. Holtom, and X. S. Xie, Phys. Rev. Lett. 82, 4142 (1999).

5. M. Hashimoto, T. Araki, and S. Kawata, Opt. Lett. 25, 1768 (2000).

6. E. O. Potma, D. J. Jones, J.-X. Cheng, X. S. Xie, and J. Ye, Opt. Lett. 27, 1168 (2002).

7. C. L. Evans, E. O. Potma, M. Puoris'haag, D. Coté, C. P. Lin, and X. S. Xie, Proc. Natl. Acad. Sci. USA 102, 16807 (2005).

8. O. Burkacky, A. Zumbusch, C. Brackmann, and A. Enejder, Opt. Lett. 31, 3656 (2006).

9. H. N. Paulsen, K. M. Hilligsøe, J. Thøgersen, S. R. Keiding, and J. Larsen, Opt. Lett. 28, 1123 (2003).

10. E. R. Andresen, V. Birkedal, J. Thøgersen, and S. R. Keiding, Opt. Lett. 31, 1328 (2006).

11. E. R. Andresen, C. K. Nielsen, J. Thøgersen, and S. R. Keiding, Opt. Express 15, 4848 (2007).

12. K. Kieu, B. G. Saar, G. R. Holtom, X. S. Xie, and F. W. Wise, Opt. Lett. 34, 2051 (2009).

13. K. Tamura, E. P. Ippen, H. A. Haus, and L. E. Nelson, Opt. Lett. 18, 1080 (1993).

14. F. Tauser, A. Leitenstorfer, and W. Zinth, Opt. Express 11, 594 (2003).

15. F. Adler, A. Sell, F. Sotier, R. Huber, and A. Leitenstorfer, Opt. Lett. 32, 3504 (2007).

16. D. von der Linde, A. Laubereau, and W. Kaiser, Phys. Rev. Lett. 26, 954 (1971).

17. K. Moutzouris, F. Adler, F. Sotier, D. Träutlein, and A. Leitenstorfer, Opt. Lett. 31, 1148 (2006).

18. G. Imeshev, M. A. Arbore, M. M. Fejer, A. Galvanauskas, M. Fermann, and D. Harter, J. Opt. Soc. Am. B 17, 1148 (2000).

19. A. Sell, G. Krauss, R. Scheu, R. Huber, and A. Leitenstorfer, Opt. Express 17, 1070 (2009).

20. F. Tauser, F. Adler, and A. Leitenstorfer, Opt. Lett. 29, 516 (2004).

21. Y. Ishigame, T. Suhara, and H. Nishihara, Opt. Lett. 16, 375 (1991). 